\title{
Protocol for New Patients
}

\section{Demographics:}

Date:

Name:

Patient $(\mathrm{Pt})$ number:

Date of birth:

Gender:

Tribe:

Religion:

Occupation:

Marital status:

Age when married:

$\mathrm{N}^{\circ}$ of children:

Head of household:

Ten-Cell leader:

Phone number of the Pt:

Address of the Pt:

How long has Pt been living in Dar es Salaam?

Contact person:

Phone number:

Witness (name and relation to patient):

Phone number of witness:

Health (recruitment) center:

Distance to health center: Means of transport:

Duration:

\section{Pork consumption:}

Do you eat pork?

$\square$ Yes $\quad \square$ No

Anybody in your family eating pork?

$\square$ Yes $\quad \square$ No

Are there free roaming pigs around?

$\square$ Yes $\quad \square$ No

Have you or any family member ever had a pork tapeworm? $\square$ Yes $\square$ No

\section{Anthelmintic treatment during last 12 months?}
$\square$ Yes
$\square$ No
$\square$ Unkown 


\section{Description of epileptic seizure(s):}

\subsection{Description by Pt and witness first in words (step by step):}

\subsection{Loss of consciousness:}

$\square$ From the beginning $\square$ After motor signs start $\quad \square$ After aura

$\square$ Clouding of consciousness $\quad \square$ No loss

\subsection{Motor activity:}

$\square$ Tonic $\quad \square$ Clonic $\quad \square$ Both

$\square$ No movements of limbs, but rolling of eyes and grinding of teeth

$\square$ No movements at all

\subsection{Side of limb movements:}

$\square$ Only left side $\quad \square$ Only right side $\quad \square$ Both sides

4.5 Fitting for how long?

Ever fitted >than 30 min? $\square$ Yes $\square$ No

If yes, give time?

\subsection{Supportive signs:}
$\square$ Froth from mouth
$\square$ Tongue/lip bite

$\square$ Urine/faecal incontinence

\subsection{Contracted any injuries during fits? $\square$ Yes $\square$ No}

What kind of injury (burn, bruise etc.?, where?, size in $\mathrm{cm} ?$ )

When contracted?

Medical attention sought? $\square$ Yes $\square$ No

Where? $\square$ Hospital $\square$ Dispensary $\square$ Traditional healer

Action taken? $\square$ Surgery $\square$ Dressing

$\square$ Drugs, which?

$\square$ Other:

\subsection{Reorientation (postictal) phase present? $\square$ Yes $\square$ No}

How long?

Description of reorientation phase: 
$\square$ Aggression $\quad \square$ Confusion $\square$ Sleeping/sleepy

\subsection{Are epileptic seizures always the same? $\square$ Yes $\square$ No}

If different, describe and make clear to which type of seizure(s) the information in the questionnaire refers to?

\section{Frequency:}

When did seizures start?

Date: Last seizure:

How many seizures in total:

How many seizures within one month:

Does the Pt have multiple seizures within one day (how many):

If yes, is Pt waking up in between?

What was Pt doing just before first seizure?

Was Pt healthy just before the first seizures?

When during the day are most of the seizures?
$\square$ Waking or the hour thereafter
$\square$ Morning $\square$ Afternoon
$\square$ Anytime during the day
$\square$ Evening $\quad \square$ Night while asleep
$\square$ Anytime at day or night
$\square$ Other:

\section{Prodromi/Aura:}

$\square$ Seizures start suddenly $\square$ Pt knows when they are coming (premonition)

Description of sensation:

How long before?

Focal signs like twitching of arm/leg before losing consciousness?
$\square$ Yes
$\square$ No

How long before?

\section{Association of other illnesses in relation to first epileptic seizure:}
Any illness prior to first seizure
$\square$ Yes
$\square$ No

Details (what illness, how long before first seizure):

\section{Precipitants: $\square$ Yes $\quad \square$ No}

Any precipitants that bring seizures on? 

$\square$ Fever
$\square$ Alcohol
$\square$ Menstruation
$\square$ Sleep
$\square$ Emotional stimuli
$\square$ Other:

\section{Past medical history (before the seizures started):}

9.1 Description of severe/chronic illnesses in the past (type, date, action taken, permanent condition):

9.2 Does/did the Pt drink alcohol (age when started, age when stopped, how many days per week, how much does Pt drink, how often does Pt get drunk):

9.3 Febrile fits (FF) in the past (how many episodes, age, how many fits per episode per $24 \mathrm{hrs,} \mathrm{FF} \mathrm{unilateral/bilateral,} \mathrm{average} \mathrm{time} \mathrm{of} \mathrm{FF,} \mathrm{sequelae):}$

9.4 Accidents, injuries or traumata (type, date, action taken, permanent condition):

9.5 Hospital admissions (what hospital, reason, date, time of stay):

10. Episodes of severe progressive headache within last months?
$\square$ Yes
$\square$ No
$\square$ Unkown

\section{Past psychiatric history: $\square$ Yes $\square$ No}

Description of psychiatric problems in the past and at present:
$\square$ Depression
$\square$ Mental retardation
$\square$ Behavioural problems
$\square$ Dementia
$\square$ Psychotic episodes
$\square$ Other:

Severity:

$\square$ Mild $\square$ Moderate

$\square$ Severe

Since when:

Action taken:

\section{Drug history:}
12.1 Pt currently on treatment (Tx):
$\square$ Yes
$\square$ No

Details: 
12.2 Has the Pt ever been on AED? $\square$ Yes $\quad \square$ No

Details (drugs, dosage, how long):
12.3 Herbal Tx tried?
$\square$ Yes
$\square$ No

Description (what ingredient, route, when started, for how long taken, any help, side effects):

\subsection{Scarifications performed? $\square$ Yes $\quad \square$ No}

Description (when, where, manipulation of wound, any help, side effects):

\section{Family history:}
Any known cases of epilepsy/seizure(s) in family? $\square$ Yes
$\square$ No

Who?

Description of seizure(s) (age at first, last seizure, frequency, precipitant(s)):

Any known cases of other neurological/mental illnesses in family?

$\square$ Yes $\quad \square$ No

Who?

What illness?

\section{Birth history and perinatal history:}

14.1 Was mother healthy during pregnancy? $\square$ Yes

Type of illness, medication taken:

\subsection{Description of delivery:}
$\square$ Hospital
$\square$ Dispensary
$\square$ Home
$\square$ On term
$\square$ Preterm
$\square$ Spontaneous vaginal delivery $\square$
Caesarean section
$\square$ Assisted delivery (forceps etc.)

\subsection{Labour:}
$\square$ Prolonged
$\square$ Precipitated
$\square$ Normal

Duration in hours:

\subsection{Baby:}



Baby crying soon?
$\square$ Yes
$\square$ No
Baby sucking well?
$\square$ Yes
$\square$ No
Baby blue?
$\square$ Yes
$\square$ No
Baby yellow?
$\square$ Yes
$\square$ No

14.5 Other problems (resuscitation, incubator, oxygen supply, neonatal seizures etc.):

\subsection{Milestones:}

Delayed/normal?

\begin{tabular}{|l|l|l|l|l|l|l|}
\hline Head control & Turning & Sitting & Crawling & Standing & Walking & Talking \\
\hline & & & & & & \\
\hline
\end{tabular}

Delayed since when: since birth

Special event:

14.7 Neonatal seizures (age, frequency, any associated symptoms/signs):

\section{School attendance:}

Did/does Pt go to school? $\square$ Yes

$\square$ No, reason:

Age started?

Level achieved?

Regular attendance? $\square$ Yes

$\square$ No, reason:
$\square$ Epilepsy
$\square$ Financial $\square$ Social
$\square$ Other:

Drop out? $\square$ Yes

$\square$ No, reason:
$\square$ Epilepsy
$\square$ Financial
$\square$ Social
$\square$ Other:

\section{Work:}

Before first fit: Type of work? 
Hours of work?

Since fits started: Type of work?

Hours of work?

Since Tx started: $\quad$ Type of work?

Hours of work?

If there is a difference, give reason:

After a fit: Days lost due to fits:

Days with impaired work:

On days with impaired work Pt works: $\quad \square<50 \% \quad \square>50 \%$

\section{Neurological signs:}

\section{Mental state:}

$\begin{array}{lll}\text { Appearance: } & \square \text { Normal } & \square \text { Abnormal: } \\ \text { Behaviour: } & \square \text { Normal } & \square \text { Abnormal: } \\ \text { Cooperation: } & \square \text { Normal } & \square \text { Abnormal: } \\ \text { Affect: } & \square \text { Normal } & \square \text { Abnormal: } \\ \text { Speech: } & \square \text { Normal } & \square \text { Abnormal: } \\ \text { Mood: } & \square \text { Normal } & \square \text { Abnormal: } \\ \text { Thought: } & \square \text { Normal } & \square \text { Abnormal: } \\ \text { Cognition: } & \square \text { Normal } & \square \text { Abnormal: }\end{array}$

\section{Impression:}

\subsection{Kind of seizures:}

$\square$ Symptomatic (provoked) seizures:

$$
\begin{aligned}
\text { Cause: } & \square \text { Alcohol } \quad \square \text { Fever } \quad \square \text { Malaria } \\
& \square \text { Other: }
\end{aligned}
$$

$\square$ Febrile seizures

$\square$ Unprovoked single seizure

$\square$ Pseudoseizures

19.2 Type of epileptic seizures (see explanatory notes):

$\square$ Generalized seizures within a specific age range 
$\square$ Generalized seizures outside a specific age range

$\square$ Generalized seizures with brain damage

$\square$ Generalized seizures with focal signs

$\square$ Simple partial seizures

$\square$ Two different seizure types, which?

$\square$ Unclassified seizures

$\square$ Other types of seizures (juvenile myoclonic, Salaam attacks, absences, complex partial):

\subsection{Loss of consciousness only:}

$\square$ Provoked, cause:

$\square$ Unprovoked
19.4 Syncope and pre-syncope:
$\square$ Yes
$\square$ No
19.5 Non-epileptic myoclonus:
$\square$ Yes
$\square$ No

\subsection{Psychiatric diagnosis:}

$\square$ Yes, specify:

$\square$ No

20. Plan:
$\square$ Phenobarbitone
Dose:
Tabs given:
$\square$ Carbamazepine
Dose:
Tabs given:
$\square$ Valproate
Dose:
Tabs given:

Health education done?

$\square$ Yes

$\square$ No

Next appointment:

Comments:

\section{Explanatory notes to types of epileptic seizures:}

\section{Generalized types of seizure:}

\section{a. Generalized seizures within a specific age range:}

$=$ primary generalized seizures that start within a specific age group (mainly between 6 and 25 years old). Seizures in this group can also be termed idiopathic generalized epilepsy. 
Cause: none obvious

Prognosis: good

Investigations: keep to a minimum

b. Generalized seizures outside a specific age range:

$=$ primary generalized seizures that start outside the specific age range of most of the idiopathic generalized epilepsies where no symptoms/signs on clinical examination suggest a secondary cause.

Cause: none obvious, seizures may be "cryptogenic"

Prognosis: variable

Investigations: further investigations necessary (EEG, neuroimaging)

\section{Partial types of seizure that clinically appear as generalized seizures:}

\section{a. Generalized seizures with diffuse brain damage:}

$=$ in clinical terms these seizures start in a generalized way but diffuse brain damage with mental retardation is obvious. The large majority of patients are children.

Cause: mostly known (e.g. birth defect)

Prognosis: variable

Investigations: keep to a minimum

\section{b. Generalized seizures with focal signs:}

$=$ secondary generalized seizures with a focal start, clear unilateral seizures or focal neurological signs upon examination but without major brain damage.

Cause: becomes obvious upon further investigation

Prognosis: depends on the underlying cause

Investigations: further investigations necessary (EEG, neuroimaging)

\section{Reference:}

Winkler A, Schaffert M, Schmutzhard E (2007) Epilepsy in resource poor countries - suggestion of an adjusted classification. Epilepsia 48(5):1029-30. 